\section{A4.14 ROLE OF DIFFERENT ADIPONECTIN RECEPTORS IN ADIPONECTIN SIGNALING IN RHEUMATOID ARTHRITIS SYNOVIAL FIBROBLASTS}

doi:10.1136/annrheumdis-2013-203217.14

${ }^{1} \mathrm{~K}$ Khawaja, ${ }^{1} \mathrm{KW}$ Frommer, ${ }^{2} \mathrm{~A}$ Schäffler, ${ }^{2} \mathrm{C}$ Büchler, ${ }^{3} \mathrm{~S}$ Rehart, ${ }^{3} \mathrm{~A}$ Lehr, ${ }^{1} \mathrm{U}$ MüllerLadner, 'E Neumann. 'Department of Internal Medicine and Rheumatology, JustusLiebig-University, Giessen, Kerckhoff-Klinik, Benekestrasse 2-8, D-61231 Bad Nauheim, Germany; ${ }^{2}$ Department of Internal Medicine I, University of Regensburg, Regensburg, Germany; ${ }^{3}$ Markus-Hospital, Frankfurt, Germany

Objectives Adiponectin levels were found to be increased in the synovial fluid of rheumatoid arthritis (RA) patients suggesting a role in the pathophysiology of the disease. RA synovial fibroblasts (SF) are known to be a key cell in RA and to express adiponectin in vivo. Adiponectin exists in four isoforms, namely the globular, low molecular weight (LMW or trimer), middle molecular weight (MMW) and high molecular weight (HMW) form. These isoforms act by binding to its receptors AdipoR1, AdipoR2, PAOR3 and PAOR10, which are expressed by various cells. Subsequently, different intracellular signalling pathways are activated including AMPK, p38MAPK, and FAK. The purpose of the present study was to determine the adiponectin receptor expression and the specificity of the adiponectin receptors to the respective adiponectin isoforms mediating different signalling pathways in RASF.

Methods AdipoR1, AdipoR2, PAOR3 and PAOR10 mRNA and protein expression were analysed in RASF by Real-time PCR, Western blotting, and immunocytochemistry. AdipoR1 and AdipoR2 down-regulation by siRNA via nucleofection technology and lentiviral transduction were performed followed by signalling analysis via Western blots.

Results Real-time PCR and Western blotting results showed that cultured RASF express AdipoR1, AdipoR2, and PAOR3 but not PAOR10. This was further confirmed by immunocytochemical analysis. Transfection of siRNA specific for AdipoR1, AdipoR2 and PAOR3 via nucleofection showed successful receptor downregulation at mRNA level but no effect was observed at protein level even after 10 days, most likely due to the long receptor half-life on the cell surface. However, lentiviral transduction showed successful down-regulation of AdipoR2 at mRNA as well as at protein level after 5 weeks (approx. 5 fold). AdipoR2 knockdown in RASF showed a reduction in adiponectin-induced p38MAPK signalling.

Conclusions Our results show that RASF isolated from synovium of RA patients express the adiponectin receptors AdipoR1, AdipoR2 and PAOR3. Down-regulation of these adiponectin receptors by lentiviral transduction was found to be a more efficient technique as compared to siRNA transfection, which will facilitate to study their role in signalling. Moreover, the induction of the p38MAPK pathway by adiponectin seems to be dependent on AdipoR2.

Funding This work was supported by the German research society (NE1174/8-1).

\section{A4.15 SERUM LEVELS OF VISFATIN AND B-CELL ACTIVATING FACTOR OF THE TNF FAMILY CORRELATE WITH DISEASE ACTIVITY IN PATIENTS WITH MYOSITIS}

doi:10.1136/annrheumdis-2013-203217.15

'H Hulejová, 'O Kryštưfková, 'H Mann, 'M Klein, '2K Pavličková, '2J Zámečník, 'L Šenolt, $1 \mathrm{~J}$ Vencovský. ${ }^{1}$ Institute of Rheumatology, Experimental and Clinical Rheumatology, First Faculty of Medicine, Charles University in Prague, Czech Republic; ${ }^{2} 2^{\text {nd }}$ Medical School and University Hospital Motol, Department of Pathology and Molecular Medicine, Charles University, Prague, Czech Republic

Background and Objectives Visfatin is an adipocytokine that supports B-lymphocyte precursor maturation, and also takes part in regulation of inflammation. Anti-histidyl-tRNA syntethase antibodies (anti-Jo-1) are the most frequent myositis specific autoantibodies. We have shown increased serum levels of B-cell activating factor of the TNF family (BAFF) in dermatomyositis (DM) and anti-Jo-1-positive polymyositis (PM) patients and its association with disease activity. Here we evaluated serum levels of visfatin in anti-Jo-1-positive PM/DM patients, its expression in muscle tissue and investigated potential relations between visfatin, BAFF disease activity and anti-Jo-1 autoantibody levels.

Material and Methods ELISA was used for detection of visfatin, BAFF and anti-Jo-1 serum samples in patients with PM $(n=27)$, $\mathrm{DM}(\mathrm{n}=11)$ and in 25 age and sex matched healthy controls. Paired samples from two different time points of sixteen patients were available. Disease activity was evaluated by myositis disease activity assessment visual analogue scales (MYOACT) and by serum levels of creatine phosphokinase (CPK), aminotranspherases (ALT, AST), lactate dehydrogenase (LDH) and myoglobin. Visfatin was detected by immunohistochemistry in muscle tissues of PM/DM patients $(n=5 / 5)$ and compared with non-inflammatory control muscle tissues from patients with myasthenia gravis $(n=5)$.

Results Serum visfatin and BAFF levels were significantly higher in myositis patients (medians 1.9 and $1.4 \mathrm{pg} / \mathrm{l}$ ) compared to healthy controls (1.3 and $1.0 \mathrm{pg} / \mathrm{l} ; \mathrm{p}<0.02$ and $\mathrm{p}=0.003$ ) and were associated with clinical muscle activity $(r s=0.39 ; p<0.02$ and $r s=0.34$ $p=0.04$ ). Trend for correlation of both visfatin and BAFF with the global disease activity was present. Serum levels of visfatin were associated with LDH ( $r s=0.39, p<0.02)$, whereas BAFF correlated with CPK, myoglobin and AST ( $r s=0.51,0.57$ and $0.39 ; \mathrm{p}<0.05$ for all). Positive correlation between visfatin and BAFF serum levels was found in patients with myositis ( $r s=0.44 ; p<0.01$ ) but was negative in healthy controls ( $r s=-0.54 ; \mathrm{p}<0.001$ ). No association between visfatin and anti-Jo-1 autoantibody levels was found while BAFF positively correlated with anti-Jo-1 levels ( $r s=0.85 ; p=0.001$ ). Visfatin levels decreased significantly over time $(p=0.01)$, while the decrease of BAFF was not significant. Visfatin expression was present in endomysial and perimysial inflammatory infiltrates of muscle tissues from patients with PM/DM compared with no expression in controls.

Conclusions These results demonstrate that serum levels of visfatin, similarly to BAFF, associate with disease activity in patients with myositis. Increased visfatin levels and expression in inflamed muscle tissues support its potential role in the pathogenesis of idiopathic inflammatory myopathies.

Acknowledgement This study was supported by Research Project No. 00023728 and Internal Grant Agency of Ministry of Health of the Czech Republic NT/12438-4.

\section{A4.16 HIGH MOLECULAR WEIGHT ADIPONECTIN AND TUMOR NECROSIS FACTOR-A INFLUENCE RHEUMATOID ADIPOSE- DERIVED MESENCHYMAL STEM CELLS FUNCTION}

doi:10.1136/annrheumdis-2013-203217.16

U Skalska, W Maslinski, E Kontny. Institute of Rheumatology, Department of Pathophysiology and Immunology, Warsaw, Poland

Background and Objectives Adipose-derived mesenchymal stem cells (ASCs) are promising target in autoimmune diseases therapy. Adipocytokines are known to influence immune system and their role in rheumatoid arthritis (RA) is intensively studied. Objectives of our work were:

1. to investigate whether leptin, low (LMW) and high (HMW) molecular weight adiponectin isoforms and TNF affect IL-6, IL-8, VEGF and TGF $\beta$ secretion by RA-ASCs.

2. to determine if conditioned media from RA-ASCs treated with above mentioned adipocytokines, influence function of rheumatoid FLS: IL- 6 and MMP-3 secretion, proliferation and apoptosis.

Materials and Methods Articular adipose tissue and synovial membrane were obtained from 18 RA patients during total knee 
joint replacement surgery. ASCs were isolated and cultured with/ without human recombinant leptin, TNF, adiponectin (LMW, HMW) and IFN $\gamma$. After $24 \mathrm{~h}$, secretion of cytokines was measured by ELISA. Conditioned media from ASCs cultures were used to stimulate FLS. After stimulation, FLS proliferation and apoptosis was determined (by incorporation of $\mathrm{BrdU}$ and flow cytometry, respectively). Cytokine concentration in FLS culture supernatants was determined by ELISA. The Wilcoxon signed-rank test was used for statistical analysis.

Results HMW adiponectin enhanced IL-6, IL-8, VEGF $(p<0.001)$ and TGF $\beta(p<0.01)$ production. LMW adiponectin increased secretion of IL- 6 and VEGF $(p<0.001)$, but its influence was much weaker than HMW's. TNF was the most potent in stimulating IL-6 and IL-8 production by ASCs $(\mathrm{p}<0.001)$. The influence of adipocytokine treated-ASC-conditioned media on FLS was assesed by comparison with two controls: untreated ASC-conditioned medium and control FLS medium. ASC-conditioned medium treated with HMW caused down-regulation of IL- 6 secretion by FLS comparing to both controls $(p<0.05)$. TNF-treated ASC-conditioned medium induced significant increase in MMP-3 production by FLS comparing to both controls $(p<0.05)$. Proliferation of FLS was up-regulated after untreated ASC-conditioned medium $(p<0.01)$ and this was partly reversed by HMW and TNF-treated ASC-conditioned media $(p<0.05)$. HMW-treated ASC-conditioned medium increased percentage of FLS in late apoptosis $(\mathrm{p}<0.05)$.

Conclusions HMW and TNF seem to be the most potent in altering RA-ASCs properties. Leptin had no effect and LMW exerted only slight effect. HMW was able to up-regulate factors thought to mediate ASCs immunosupression (TGF $\beta$, IL-6). In addition, its effect on FLS shows that this adipokine may exert positive effect on immunosupressive ASCs function. However, because HMW upregulated also proangiogenic cytokines (IL-8, VEGF), it is difficult to determine its real impact on RA-ASCs. TNF role in modulating RAASCs also need to be elucidated.

Funding Grant No. N/NZ5/00932, National Science Center, Poland.

\section{B cells and autoimmunity}

\section{A5.1 ABNORMAL CALCIUM INFLUX IN T AND B LYMPHOCYTES FROM SYSTEMIC LUPUS ERYTHEMATOSUS PATIENTS IS RELATED TO STIM-1 OVER-EXPRESSION}

doi:10.1136/annrheumdis-2013-203219.1

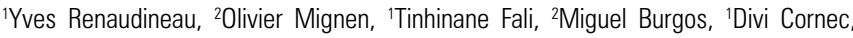
'Sandrine Jousse, 'Alain Saraux, 'Jacques-Olivier Pers. 'EA2216 Immunology, Pathology and Immunotherapy, Brest University and Brest Medical School Hospital, Brest, France; 2INSERM U613, Faculty of Medicine, Brest, France

Background and Objectives Recently described, the molecule STIM1 (stromal interaction molecule 1) acts as a key mediator of calcium influx by controlling cell proliferation after antigen stimulation, Erk phosphorylation, cytokine production and apoptosis. Although STIM1 mutations have been associated with severe immunodeficiency, no study has focused on the STIM1 molecule in autoimmune diseases.

Materials and Methods $\mathrm{T}$ and $\mathrm{B}$ lymphocytes, purified by negative selection from peripheral blood of patients with systemic lupus erythematosus (SLE, $\mathrm{n}=11$ ), rheumatoid arthritis (RA, $n=7)$, primary Sjögren's syndrome ( $\mathrm{pSS}, \mathrm{n}=11)$ and healthy controls $(\mathrm{HC}, \mathrm{n}=12$ ) were tested by flow cytometry and Western blotting to determine the expression of STIM1. Video microscopy using specific probes has been used to assess intracellular calcium levels.
Results T cells from peripheral blood of HC express more STIM1 molecules (mean fluorescence intensity (MFI) $3.42 \pm 0.13$ ) than $B$ cells (MFI $2.18 \pm 0.20, \mathrm{P}<0.01$ ). In B lymphocyte subpopulations, the expression of STIM1 is 2 times higher in $\mathrm{CD} 24^{\text {high }} \mathrm{CD} 38^{\text {high }}$ transitional B cells (MFI $4.83 \pm 0.63$ ) compared with CD24 ${ }^{\text {low }} \mathrm{CD} 38^{\text {low }}$ mature $B$ cells (MFI $2.47 \pm 0.15, \mathrm{P}<0.01$ ) and $\mathrm{CD} 24^{\text {high }} \mathrm{CD} 38^{\text {low }}$ memory B cells (MFI $3.64 \pm 0.42, \mathrm{P}<0.05$ ). The expression of STIM1 in T and B lymphocytes from patients with RA and pSS was similar to HC.

An highest calcium influx and a constitutive Erk phosphorylation characterise $\mathrm{T}$ and $\mathrm{B}$ cells from SLE patients when compared with HC and disease controls. As suspected, STIM1 is overexpressed in SLE, when compared with $\mathrm{HC}$ and this expression is similar between T cells (MFI $8.70 \pm 0.87$ ) and B cells (MFI $9.00 \pm$ 1.08). Within B cell subsets, STIM1 expression is 3.4 fold highest in transitional SLE B cells (MFI: $16.25 \pm 2.18, P<0.001$ ) than in transitional HC B cells and 2.3 fold highest than in mature SLE B cells (MFI: $7.1 \pm 1.53$ ) and memory SLE B cells (MFI: $9.51 \pm 2.01$ ). Western blotting results confirm the highest expression of STIM1 in SLE. Transient transfection of STIM1-targeting siRNAs was shown to restore the calcium influx and decrease Erk phosphorylation. Of particular note, the associations of $\mathrm{CpG} / \mathrm{anti-IgM} \mathrm{Ab}$ and $\mathrm{CpG}$ /anti-CD40 $\mathrm{Ab}$ are effective to induce STIM1 expression. Finally, STIM1 level was not correlated with the SLE disease activity index (SLEDAI) and autoantibodies (ANA, anti-dsDNA, anti-SSA/Ro).

Conclusions These results suggest that the differential expression of STIM1 may be an important factor in the process of lymphocyte self-reactivity in SLE, which opens new pathophysiological and therapeutic perspectives.

\section{A5.2 ACCUMULATION OF CIRCULATING AUTOREACTIVE NAÏVE B CELLS REVEAL DEFECTS OF EARLY B CELL TOLERANCE CHECKPOINTS IN PATIENTS WITH SJÖGREN'S SYNDROME}

doi:10.1136/annrheumdis-2013-203219.2

'Elisa Corsiero, 'Nurhan Sutcliffe, ${ }^{2}$ Hedda Wardemann, 'Costantino Pitzalis, 'Michele Bombardieri. 'Centre for Experimental Medicine \& Rheumatology, William Harvey Research Institute, Barts and The London School of Medicine \& Dentistry, Queen Mary University of London, John Vane Science Centre, Charterhouse Square, London EC1M 6BO, UK; ${ }^{2}$ Max Planck Institute for Infection Biology, 10117 Berlin, Germany

Background and Objectives Sjögren's syndrome (SS) is an autoimmune disease characterised by high affinity circulating autoantibodies and peripheral $\mathrm{B}$ cell disturbances with predominance of naive and reduction of memory $B$ cells. The stage at which errors in $\mathrm{B}$ cell tolerance checkpoints accumulate in SS is unknown. Here we determined the frequency of self- and poly-reactive B cells in the circulating naïve compartment of SS patients.

Materials and Methods Single CD27-IgD + B cells were sorted by FACS from peripheral blood of SS patients and healthy donors (HD). RNA was used to amplify Ig VH and VL genes and PCR products were cloned and expressed as recombinant monoclonal antibodies displaying identical specificity of the original B cells. Recombinant antibodies were tested towards different antigens to determine the frequency of autoreactive and polyreactive clones.

Results 66 recombinant antibodies were generated from naïve B cells of 4 SS patients and compared to 45 clones from $2 \mathrm{HD}$. Analysis of the $\mathrm{VH}$ and VL gene usage showed no significant differences between SS and HD. Conversely, we observed accumulation of circulating autoreactive naïve B cells in SS as demonstrated by increased reactivity towards Hep2 cells (43.1\% SS versus $25 \% \mathrm{HD}$ ) and ENA (19.6\% SS clones versus none). Among ENA+ clones, 6 displayed reactivity towards Ro/SSA and/or La/SSB. 